\title{
Philosophiques
}

\section{La métaphysique de la nature à l'Académie de Berlin}

\section{Christian Leduc}

Volume 42, numéro 1, printemps 2015

La philosophie à l'Académie de Berlin au XVIII ${ }^{\mathrm{e}}$ siècle

URI : https://id.erudit.org/iderudit/1032215ar

DOI : https://doi.org/10.7202/1032215ar

Aller au sommaire du numéro

Éditeur(s)

Société de philosophie du Québec

ISSN

0316-2923 (imprimé)

1492-1391 (numérique)

Découvrir la revue

Citer cet article

Leduc, C. (2015). La métaphysique de la nature à l'Académie de Berlin. Philosophiques, 42(1), 11-30. https://doi.org/10.7202/1032215ar

\section{Résumé de l'article}

Dans le présent article, je montre que Maupertuis et Euler proposent une conception contrastée de la métaphysique de la nature. Il s'agit principalement pour eux de repositionner la cosmologie par rapport aux sciences de la nature. $\mathrm{Au}$ lieu de considérer la métaphysique comme étant au fondement des théories scientifiques, comme le supposent Descartes, Wolff et, d'une certaine manière, Kant, ou simplement d'interdire l'idée même d'une cosmologie, comme le stipulerait à la même époque d'Alembert, Maupertuis et Euler, renversent l'ordre des disciplines pour donner aux sciences physiques une primauté sur la métaphysique. Ce repositionnement entraîne évidemment plusieurs questions : d'abord, de quelle manière les théories scientifiques peuvent-elles valider ou infirmer un principe métaphysique ? Plus important encore, quel rôle incombe à la métaphysique de la nature, étant donné que les sciences possèdent non seulement une autonomie théorique, mais même une priorité sur les réflexions ontologiques. Il semblerait d'ailleurs que la métaphysique se réduise, pour ces deux philosophes, au seul domaine du cosmologique, en particulier aux questions qui concernent la force et le principe de moindre action, au statut ontologique de l'espace et du temps et aux déterminations de la matière que sont l'étendue, l’inertie, l’impénétrabilité et le mouvement.
Ce document est protégé par la loi sur le droit d'auteur. L’utilisation des services d’Érudit (y compris la reproduction) est assujettie à sa politique d'utilisation que vous pouvez consulter en ligne.

https://apropos.erudit.org/fr/usagers/politique-dutilisation/ 


\title{
La métaphysique de la nature à l'Académie de Berlin
}

\author{
CHRISTIAN LEDUC \\ Université de Montréal \\ christian.leduc.1@umontreal.ca
}

\begin{abstract}
RÉSUMÉ. - Dans le présent article, je montre que Maupertuis et Euler proposent une conception contrastée de la métaphysique de la nature. II s'agit principalement pour eux de repositionner la cosmologie par rapport aux sciences de la nature. Au lieu de considérer la métaphysique comme étant au fondement des théories scientifiques, comme le supposent Descartes, Wolff et, d'une certaine manière, Kant, ou simplement d'interdire l'idée même d'une cosmologie, comme le stipulerait à la même époque d'Alembert, Maupertuis et Euler, renversent l'ordre des disciplines pour donner aux sciences physiques une primauté sur la métaphysique. Ce repositionnement entraîne évidemment plusieurs questions: d'abord, de quelle manière les théories scientifiques peuvent-elles valider ou infirmer un principe métaphysique? Plus important encore, quel rôle incombe à la métaphysique de la nature, étant donné que les sciences possèdent non seulement une autonomie théorique, mais même une priorité sur les réflexions ontologiques. II semblerait d'ailleurs que la métaphysique se réduise, pour ces deux philosophes, au seul domaine du cosmologique, en particulier aux questions qui concernent la force et le principe de moindre action, au statut ontologique de l'espace et du temps et aux déterminations de la matière que sont l'étendue, l'inertie, l'impénétrabilité et le mouvement.
\end{abstract}

ABSTRACT. - In this paper, I show that Maupertuis and Euler offer a contrasting conception of metaphysics of nature. It consists mainly for them in repositioning cosmology in relation to natural sciences. Instead of considering metaphysics to be at the foundation of scientific theories, as was assumed by Descartes, Wolff, and, in a certain way, Kant, or simply prohibiting the very idea of a cosmology, as d'Alembert would stipulate at the same period, Maupertuis and Euler invert the order of disciplines to give priority to physical sciences over metaphysics. This repositioning leads of course to several questions: first, how does scientific theories validate or deny metaphysical principles? Even more important, which role falls to metaphysics of nature given that sciences not only possess a theoretical autonomy, but also a priority over ontological reflections? Besides, it seems that metaphysics would be reduced, for these two philosophers, to the realm of cosmology alone, in particular to questions concerning force and the principle of least action, the ontological status of space and time, and the first constituents of matter, such as extension, inertia, impenetrability, and motion.

Dans les Metaphysische Anfangsgründe der Naturwissenschaft, Kant défend une conception de la métaphysique de la nature qui s'ajuste, comme il est bien connu, au cadre d'une philosophie transcendantale. La science de la nature doit comporter une partie pure, dont les principes sont a priori et nécessaires, et mènent à une certitude apodictique, laquelle servira de 
fondement aux principes physiques ou psychologiques qui, eux, sont d'origine empirique. L'un des aspects importants de cette doctrine est que la validité d'une métaphysique de la nature se mesure d'une certaine manière à sa capacité d'application à des objets mathématisables. Une science de la nature porte sur des objets déterminés dont la possibilité n'est pas seulement conceptuelle, mais aussi construite in concreto à partir des données pures de l'intuition, conditions d'établissement des mathématiques. J'aimerais cependant insister sur un autre aspect qui concerne le positionnement de la métaphysique de la nature dans les Anfangsgründe. La conception kantienne s'ancre en réalité dans une longue tradition qui interprète la métaphysique de la nature en tant qu'instituant un fondement pour les sciences naturelles:

Une science de la nature qui s'appelle ainsi à proprement parler présuppose une métaphysique de la nature; car des lois, c'est-à-dire des principes de la nécessité de ce qui appartient à l'existence d'une chose, se rapportent à un concept qu'on ne peut construire parce que l'existence ne peut se représenter dans aucune intuition a priori. C'est pourquoi la science de la nature proprement dite suppose la métaphysique de la nature².

Le rapport entre la métaphysique de la nature et la science de la nature en est un de priorité où la deuxième suppose la première. Bien qu'elle se situe dans un contexte transcendantal qui lui est propre, cette conception reproduit une articulation assez commune à l'époque moderne entre la métaphysique et la physique: la physique, comme science expérimentale, nécessite une réflexion métaphysique a priori qui analyse les concepts se rapportant à ses objets. Kant partage ainsi avec certains philosophes modernes une manière de concevoir l'articulation entre métaphysique de la nature et science naturelle, en particulier avec Descartes et Wolff. La métaphysique, telle que positionnée par Descartes dans la préface à l'édition française des Principes de la philosophie, est bien connue en tant qu'elle représente les racines d'une forme arborescente, laquelle illustre l'organisation des savoirs scientifiques et son fondement dans la philosophie première ${ }^{2}$. Au sein des Lumières allemandes, c'est chez Wolff qu'on trouve une définition similaire des rapports entre métaphysique et physique: dans son Discursus proeliminaris, Wolff soutient que la métaphysique et la cosmologie générale, laquelle a le monde et les corps totaux pour objets, démontrent les premiers principes qui sont nécessaires à la physique ${ }^{3}$. La physique tire ainsi ses principes de l'ontologie et de la cosmologie. Il y aurait même une priorité méthodologique de la métaphysique sur la physique d'après Wolff:

1. Gesammelte Schriften, hrsg. von der Königlich preußischen Akademie der Wissenschaften, Berlin, G. Reimer, I902-, IV, p. 469, trad. par J. Gibelin, Paris, Vrin, I97I, p. IO.

2. Euvres de Descartes, éd. par C. Adam et P. Tannery (I897-I9I3), présentation par B. Rochot et P. Costabel, Paris, Vrin, I964-I974 (abrév. AT), IX-b, p. I4.

3. Discursus proeliminaris, III, 94, in Gesammelte Werke, hrsg. von J. École u.a., Olms, Hildesheim, I965-, II, I, I, p. 42. 
De ce qui a été dit, il est patent qu'il faut disposer la Métaphysique avant la Physique, si l'on doit traiter celle-ci de manière démonstrative. En effet, s'il faut traiter la Physique de manière démonstrative, il faut tirer ses principes de la Métaphysique ${ }^{4}$.

Pour le dire succinctement, la métaphysique, pour Descartes, Wolff et Kant, constituerait un prérequis théorique nécessaire à l'élaboration complète et certaine de la physique. La légitimation de la métaphysique se comprend en ce qu'elle est présupposée, dans ses principes, par les sciences de la nature. Évidemment, le contenu respectif de la métaphysique et de la physique, ainsi que l'articulation plus précise de l'une par rapport à l'autre divergent chez ces différents auteurs: force est toutefois de constater qu'ils maintiennent une conception de la métaphysique de la nature qui lui fait jouer un rôle prépondérant dans sa relation aux théories physiques, voire à l'ensemble des connaissances scientifiques.

Comme on le sait, plusieurs auteurs au XVIII ${ }^{\mathrm{e}}$ siècle sont très critiques de cette définition de la métaphysique en ce qu'elle serait nécessaire à l'élaboration de la physique. La pensée d'inspiration newtonienne et lockienne interdira très souvent à la métaphysique ou à la cosmologie générale d'établir des principes spéculatifs sur la base desquels la physique pourra s'ériger. Voltaire, Condillac ou d'Alembert, pour ne citer que ceux-là, ont condamné cet usage de la métaphysique. Dans le cas qui nous concerne, une métaphysique de la nature non seulement serait inutile pour eux, mais mènerait à des incertitudes qui nuisent aux sciences physiques. Le seul domaine auquel devrait se borner la métaphysique, du moins pour Condillac et d'Alembert, est celui de l'analyse des capacités cognitives de l'esprit ${ }^{5}$. Bien entendu, cela veut également dire que les sciences naturelles et les mathématiques, et non la métaphysique, peuvent seules prétendre énoncer les lois du monde physique. Les sciences de la nature institueraient des lois et des principes physiques indépendamment de la métaphysique, et sans que celle-ci n'intervienne dans le raisonnement et l'expérimentation qui leur sont propres.

4. Ibid, III, 95, p. 43, trad. par T. Arnaud, W. Feuerhahn, J.-F. Goubet et J.-M. Rohrbasser, Paris, Vrin, 2006, p. I35.

5. Condillac, Essai sur l'origine des connaissances humaines, in Cuvres philosophiques de Condillac, Paris, PUF, I947, I, p. 3 ; d'Alembert, Essai sur les éléments de philosophie, Paris, Fayard, I986, p. 39. Dans le Discours préliminaire de l'Encyclopédie, d'Alembert est toutefois beaucoup moins sévère à l'endroit de la métaphysique. Il reconnaît même une fonction à une physique générale qui porte sur les premiers principes, nommée métaphysique des corps: "La Science de la nature n'est autre que celle des corps. Mais les corps ayant des propriétés générales qui leur sont communes, telles que l'impénétrabilité, la mobilité, et l'étendue, c'est encore par l'étude de ces propriétés que la Science de la nature doit commencer: elles ont, pour ainsi dire, un côté purement intellectuel par lequel elles ouvrent un champ immense aux spéculations de l'esprit, et un côté matériel et sensible par lequel on peut les mesurer. La spéculation intellectuelle appartient à la Physique générale, qui n'est proprement que la Métaphysique des corps; et la mesure est l'objet des Mathématiques, dont les divisions s'étendent presqu'à l'infini ", Paris, Vrin, 2000, p. II3. 
De manière générale, on pourrait par conséquent supposer que, à l'époque des Lumières, deux conceptions principales de la métaphysique de la nature s'opposent l'une à l'autre: la première, défendue notamment par Wolff et Kant, selon laquelle la physique a besoin de principes a priori et premiers qui sont à chercher dans une métaphysique de la nature, particulièrement en ce qui concerne la structure de la matière et l'ordre du monde corporel; la deuxième, chez des philosophes comme Condillac et d'Alembert, rejetant ce rôle assigné à la cosmologie et reconnaissant à la physique une autonomie théorique entière qui possèderait dès lors ses critères de validation propres, plus précisément la confirmation empirique et le calcul mathématique. Nul besoin de recourir selon eux à une ontologie dans l'élaboration des principes physiques; au contraire, ceux-ci doivent essentiellement prendre appui sur des méthodes antimétaphysiques et non spéculatives.

Le cas de d'Alembert est pour nous intéressant, puisque ce penseur a été un important membre et contributeur de l'Académie de Berlin. Il publia, dans les années I740 et I750, d'importants mémoires sur le calcul intégral, sur certains problèmes d'astronomie et sur la vibration des courbes ${ }^{6}$. Or l'interprétation de la métaphysique qu'on trouve chez d'Alembert à la même époque, en particulier dans son Essai sur les Éléments de philosophie de I759, pourrait sembler représentative de la position des philosophes d'obédience newtonienne à l'Académie de Berlin dans les mêmes années. Pensons surtout à Maupertuis, qui fut président de I 746 à I 757, et à Euler, membre influent et prolifique qui résida à Berlin de I74I à I766. Il est vrai que les deux sont très critiques des métaphysiques leibnizienne et wolffienne qui dominaient encore les milieux universitaires allemands à la même période. Dans ses Lettres, Maupertuis s'en prend d'ailleurs aux principaux aspects du système leibnizien, entre autres au principe de raison suffisante, à la théorie des monades et à l'harmonie préétablie . Par une approche très similaire, Euler écrivit de nombreux textes contre les thèses de Leibniz et de Wolff, en particulier ses Gedanken von den Elementen der Cörper de I746 - texte rédigé en marge du concours lancé par l'Académie de Berlin sur ce sujet qui s'attaquent à la métaphysique des monades et des éléments ${ }^{8}$. Par opposition à ces ontologies, notamment à leurs aspects cosmologiques, on pourrait ainsi croire que Maupertuis et Euler minimisent, voire éliminent la portée d'une métaphysique de la nature, à l'instar de leur collègue d'Alembert.

Par rapport aux deux conceptions de la cosmologie précédemment mentionnées, qu'on pourrait qualifier, d'une part, de fondationaliste, et,

6. Sur les contributions de d'Alembert à certains débats scientifiques de l'Académie de Berlin, voir l'important travail de J. B. Shank: The Newton War and the Beginning of the French Enlightenment, Chicago University Press, 2008, p. 449-479.

7. Cuvres, Hildesheim, Olms, II, p. 257-264.

8. Gedancken von den Elementen der Cörper, in Opera omnia, 3, 2, Leipzig, I942, p. 347-366. Sur cette critique d'Euler et le concours portant sur le système des monades, voir Christian Leduc: «Euler et le monadisme ", Studia Leibnitiana, 45/2, 2013, p. I 5 O-I69. 
d'autre part, de sceptique, il existe bien entendu d'autres manières de définir le positionnement et la fonction d'une telle discipline. L'une de celles-ci consisterait à renverser le rapport de priorité entre la métaphysique de la nature et la science de la nature pour donner à la physique la primauté sur l'ontologie. Au lieu de comprendre la première comme fournissant les principes a priori des sciences de la nature, tels que les caractéristiques de la matière ou de l'organisation du monde, il serait possible de faire de la physique la condition de légitimation et d'opérationnalisation d'une métaphysique de la nature. En d'autres mots, une métaphysique adéquate nécessiterait préalablement l'établissement de principes physiques validés par le calcul et l'expérience, et non l'inverse. Autrement, aucun principe métaphysique ne s'élaborerait de façon appropriée et déterminée, puisque ses réflexions n'auraient pas d'ancrage dans des connaissances effectives et certaines de la nature.

Or l'interprétation que je souhaite avancer consiste précisément à caractériser la conception de la métaphysique chez Maupertuis et Euler de cette dernière façon. Bien que newtoniens et antimétaphysiciens sur plusieurs aspects, Maupertuis et Euler ne croient pas qu'il soit entièrement justifié d'interdire l'élaboration d'une métaphysique de la nature. Sans revenir à une conception cartésienne ou wolffienne de la métaphysique, dite fondationaliste, ils lui réservent un rôle important dans l'étude de la nature: les principes métaphysiques de la nature s'établiraient, et ce à l'encontre de la position sceptique d'un d'Alembert ou d'un Voltaire, sur la base d'un travail scientifique préalable fournissant un ensemble de lois et de théories proprement physiques. Ensuite seulement, il serait possible de réfléchir à des questions de cosmologie qui concernent la structure de la matière et l'ordre des corps.

Je mentionnerai pour le moment un premier exemple sur lequel je reviendrai par la suite: d'après Euler, une thèse ontologique quant à la réalité de l'espace et du temps ne saurait s'établir sans l'adoption de principes mécaniques assurés. Une thèse métaphysique déterminant le caractère absolu ou relatif de l'espace et du temps serait impossible à soutenir sans l'apport des sciences naturelles qui comprennent des principes physiques certains, notamment quant aux lois du repos et du mouvement des corps ${ }^{9}$. À mon avis, comme le fait Maupertuis à la même période, Euler renverse ici non seulement certains contenus fondamentaux de la métaphysique leibnizienne et wolffienne, mais également le positionnement, qu'on trouve chez Wolff et plus tard chez Kant, entre métaphysique et physique. L'une des

9. Pour une contextualisation du mémoire d'Euler, voir Christian Leduc et Anne-Lise Rey: «L'espace dans les Lumières allemandes » in Espace et métaphysique de Gassendi à Kant, L. Peterschmitt (dir.), Paris, Hermann, 20I4, p. 285-326. Robert Disalle mentionne déjà ce changement de positionnement entre métaphysique et physique chez Euler: Understanding Space-Time. The Philosophical Development of Physics from Newton to Einstein, Camrbidge University Press, 2006, p. 50-5I. 
conséquences de cette conception serait la suivante: il semblerait que le domaine de la métaphysique se réduise à celui d'une cosmologie considérée de la sorte. Ce qui signifie somme toute deux choses: d'une part, que la physique fournit les outils conceptuels nécessaires à la détermination d'hypothèses métaphysiques; d'autre part, que, sans une telle assise, il paraît impossible d'élaborer une métaphysique, ce qui signifie qu'on en réduirait par la même occasion la portée au seul domaine cosmologique.

\section{La téléologie de Maupertuis}

Maupertuis explique peu la manière dont devrait se constituer une métaphysique, étant donné qu'il reste en général assez réticent quant à sa possibilité. Le texte où son attitude est la mieux disposée à l'égard de la métaphysique est le discours intitulé Des devoirs de l'académicien, de 1753 , dans lequel il précise de façon générale quels sont les objets et la méthode à employer dans une académie, en l'occurrence celle de Berlin. Les objets ontologiques qu'il identifie sont assez traditionnels, mais il émet toutefois une réserve quant à l'obtention de résultats certains et définitifs:

Si je vous expose ici toute la grandeur du péril des spéculations qui concernent l'Être suprême, les premières causes, et la nature des esprits, ce n'est pas, Messieurs, que je veuille vous détourner de ces recherches. Tout est permis au Philosophe, pourvu qu'il traite tout avec l'esprit philosophique, c'est-à-dire, avec cet esprit qui mesure les différents degrés d'assentiment; qui distingue l'évidence, la probabilité, le doute; et qui ne donne ses spéculations que sous celui de ces différents aspects qui leur appartient ${ }^{10}$.

Au sein d'une science qui porte sur Dieu, les principes fondamentaux de l'âme et les causes premières des choses, il faut pouvoir indiquer le type de certitude auquel appartient une vérité, ce que Maupertuis entend par l'esprit philosophique. On reconnaîtra ici sans doute l'origine lockienne de ces distinctions entre évidence, probabilité et doute, qu'on trouve dans le quatrième livre de l'Essay concerning Human Understanding. Néanmoins, l'idée d'une métaphysique générale dont les principaux objets seraient en théologie l'Être suprême, en psychologie la nature des esprits et en cosmologie les premières causes reste tout à fait envisageable. Il faut y appliquer l'esprit philosophique et déterminer les principes évidents, mais surtout les niveaux de probabilité entre les autres vérités, en écartant, le cas échéant, celles qui sont dubitables et peu probables.

La place qu'occupe la métaphysique dans le reste du corpus de Maupertuis est toutefois bien différente. Les textes qui portent sur les sujets identifiés préalablement, Dieu, l'âme et les premières causes, sont rares et contiennent très peu de principes proprement ontologiques. La plupart des textes scientifiques ou moraux de Maupertuis ne comportent d'ailleurs 
aucune réflexion véritablement métaphysique. Rappelons que la métaphysique dominante de l'époque en Allemagne, celle de Leibniz et de Wolff, fait l'objet de sévères critiques de la part de Maupertuis. La grande contribution du président de l'Académie de Berlin au domaine métaphysique se réduit pour l'essentiel à l'établissement du principe téléologique de moindre action selon lequel l'action dans les corps - qui se calcule par le produit de la masse, de la vitesse et de l'espace parcouru - est toujours la plus petite possible. De son aveu, ce principe, qui possède une grande utilité dans les sciences, est toutefois proprement métaphysique. Il existe certes une équation pour calculer les effets corporels du principe qu'Euler exprima de manière plus adéquate par la formule $\int M v d s^{11}$, de façon à ce que les conséquences physiques du principe de moindre action soient explicables par des propriétés efficientes et mathématisables. Toutefois, Maupertuis reconnaît qu'aucune loi strictement mécanique ne peut rendre intelligible la cause première qui fait en sorte que les maxima et minima de l'action sont toujours moindres. Le principe de moindre action ne relève pas de la causalité efficiente, comme les autres lois de la physique, mais de la causalité finale ${ }^{12}$, ce qui en fait bien entendu un principe de nature métaphysique. L'incursion principale de Maupertuis en métaphysique se résumerait donc à la caractérisation téléologique de son grand principe de moindre action.

Bien que le principe ait des conséquences scientifiques importantes ${ }^{13}$, j'aimerais plutôt m'attarder à sa portée ontologique. Celle-ci montre, d'après moi, que Maupertuis défend une conception de la métaphysique qui est conforme à la description que j'en ai proposée préalablement, suivant laquelle la condition d'établissement des thèses ontologiques se trouve dans la certitude des vérités scientifiques. Trois aspects me paraissent essentiels pour la présente analyse:

I) Malgré la réticence des mathématiciens de l'époque - que Maupertuis partage en bonne partie - à recourir aux causes finales, il semble que le principe général du changement ne puisse se réduire à une description de type géométrique. Maupertuis est d'accord pour dire que la multiplication des explications finales est à éviter et qu'elles ne doivent pas servir à remplacer le calcul géométrique. Dans l'Accord de différentes lois de la nature, où il est surtout question des phénomènes de la réflexion et de la réfraction, le principe de moindre action est défendu, mais avec certaines précautions quant à la portée théorique de ce genre d'explications de type téléologique:

11. Methodus inveniendi lineas curvas maximi minimive proprietate gaudentes, Lausanne/Genève, Bousquet, I744, p. 3 I I-3 I 2.

12. Accord de différentes lois de la nature, in Euvres IV, p. 20; Essai de cosmologie, in Euvres I, p. 35 .

13. À ce sujet, voir notamment l'étude de Marco Panza: «De la nature épargnante aux forces généreuses: le principe de moindre action entre mathématique et métaphysique. Maupertuis et Euler, I740-I75 I ", in Revue d'histoire scientifique 48, I995, p. 435-520. 
Le vrai principe une fois découvert, j'en déduis toutes les lois que suit la lumière, soit dans sa propagation, dans sa réflexion, ou dans sa réfraction. Je connais la répugnance que plusieurs Mathématiciens ont pour les causes finales appliquées à la Physique, et l'approuve même jusqu'à un certain point: j'avoue que ce n'est pas sans péril qu'on les introduit: l'erreur où sont tombés des hommes tels que Fermat en les suivant ne prouve que trop combien que leur usage est dangereux. On peut cependant dire que ce n'est pas le principe qui les a trompés, c'est la précipitation avec laquelle ils ont pris pour le principe ce qui n'en était que des conséquences ${ }^{14}$.

La téléologie vient ici compléter les lois de la réflexion et de la réfraction en ce qu'elle permet de décrire, par causalité finale, le mouvement de la lumière de manière exhaustive. Maupertuis reproche entre autres à Fermat de ne pas avoir suffisamment expliqué la raison téléologique de l'action de la lumière dans les phénomènes optiques. Il propose ainsi de rendre compte du fait que la lumière parcourt toujours la distance la plus courte sur la base du principe de moindre action. Dans ce texte, comme c'est le cas dans l'Essai de cosmologie, le principe est ensuite élargi à tous les phénomènes de la nature et ne s'emploie donc pas uniquement dans la description des propriétés optiques. Ces dernières ne seraient ainsi qu'un champ particulier d'application du calcul de l'action moindre. Il ne s'agit certes pas d'accroître le nombre de principes téléologiques, mais de rendre compte de ses multiples usages. En réalité, Maupertuis ne propose comme principe téléologique que l'hypothèse de la moindre action. Le principe vient compléter l'explication des phénomènes qu'il serait impossible de fonder par les seuls moyens mathématiques et physiques. D'où le recours à une explication finale et téléologique ajoutée aux descriptions et calculs physiques par causes efficientes.

2) La validité du principe de moindre action s'établirait ainsi en accord avec les lois mathématiques et physiques. C'est ici que le rapport entre métaphysique et physique est le plus important à cerner, c'est-à-dire dans l'articulation entre le principe de moindre action et les principes des sciences de la nature. Il est vrai que Maupertuis soutient à certains endroits que l'ensemble des lois de la nature se déduisent de ce premier principe, celui-ci jouant ainsi le rôle d'axiome premier à partir duquel des propositions explicatives secondes ou théorèmes sont dérivés. Dans l'Accord de différentes lois de la nature, l'explication des mouvements de la lumière est précisément dite dépendre du principe de moindre action ${ }^{15}$. L'Essai de cosmologie stipule même que les lois de la nature se déduisent de ce dernier principe:

C'est de ce principe que nous déduisons les lois du mouvement, tant dans le choc des corps durs, que dans celui des corps élastiques; c'est en déterminant bien la quantité d'action qui est alors nécessaire pour le changement qui doit 
arriver dans leur vitesse, et supposant cette quantité la plus petite qu'il soit possible, que nous découvrons ces lois générales selon lesquelles le mouvement se distribue, se produit, ou s'éteint ${ }^{16}$.

De cette manière, il semblerait que Maupertuis cautionne en quelque sorte le rapport de fondation que jouent chez certains auteurs, par exemple Wolff, les principes métaphysiques dans l'instauration des lois de la physique. À partir du principe de moindre action, il serait possible de déduire les lois de l'optique, de la mécanique, etc. ${ }^{17}$. Or, en examinant plus précisément la manière dont Maupertuis met en lien ces différentes propositions, il paraît évident que la relation entre métaphysique et physique est différente, et même contraire à une conception de type fondationaliste. D'après Maupertuis, et c'est l'interprétation que je soumettrai corrélativement à mon hypothèse de départ, il serait impossible de valider le principe de moindre action sans des confirmations mathématiques et physiques préalables. En d'autres mots, l'approbation d'une téléologie passerait nécessairement par des certitudes physiques, et non le contraire. Certains extraits viennent appuyer cette thèse d'un renversement de priorité. Par exemple, toujours dans l'Accord de différentes lois de la nature, il est affirmé que les mouvements de réflexion et de réfraction de la lumière suivent ou s'accordent avec le principe de moindre action :

Tous ces phénomènes de la réfraction s'accordent maintenant avec le grand principe que la Nature, dans la production de ses effets, agit toujours par les voies les plus simples. De ce principe suit que, lorsque la lumière passe d'un milieu dans un autre, le sinus de son angle de réfraction est au sinus de son angle d'incidence en raison inverse des vitesses qu'a la lumière dans chaque milieu $^{18}$.

En l'occurrence, la relation entre la simplicité de la production des effets qui suit une détermination téléologique et les lois de l'optique est manifestement une relation de concordance. Il paraît même impossible, malgré ce que Maupertuis dit précédemment, de simplement déduire le calcul du rapport des angles d'incidence et de réflexion ou de réfraction à partir de la thèse d'une moindre action. Maupertuis n'offre jamais une telle démonstration, et il serait à l'évidence difficile de la réaliser à l'aide du seul contenu théorique de la moindre action. Entre les deux domaines, il existe un accord,

16. Essai de cosmologie, in Cuvres I, p. 43 .

17. C'est notamment l'interprétation que donne Lambert dans les Cosmologische Briefe de la fonction de la téléologie chez Maupertuis: Philosophische Schriften, hrsg. von A. Emmel und A. Spree, Hildesheim, Olms, 2006, V, p. viii. En complément de notre analyse, voir: Michel Fichant, "Téléologie et théologie physique chez Maupertuis", in Actes de la journée Maupertuis, Paris, Vrin, I975, p. I4I-I 56; Matthias Schramm, "The Creation of the Principle of Least Action ", in Formale Teleologie und Kausalität in der Physik, Paderborn, Mentis Verlag, 2005, p. 99-II4.

18. Accord, in Euvres IV, p. I9. 
et non un rapport de fondement déductif. L'Essai de cosmologie irait par ailleurs dans le même sens, puisque la légitimité d'un usage du principe téléologique viendrait d'une application des principes de la mathématique:

J'aurai bientôt répondu à ceux qui blâment l'usage que j'ai fait des causes finales dans une matière mathématique: c'est justement ce qu'il y a de mathématique dans cette matière qui rend plus victorieuse l'application que j'y ai faite des causes finales ${ }^{19}$.

Cet extrait comprend deux aspects majeurs: d'une part, que l'intégration des causes finales dans les mathématiques, et bien entendu en physique, est faible, voire nulle si on ne peut suffisamment en justifier l'emploi. Dans les pages qui suivent, Maupertuis critique d'ailleurs les nombreuses preuves de l'existence de Dieu qui sont pour la plupart inefficaces parce que non justifiées sur le plan méthodologique ${ }^{20}$. D'autre part, ce qui autorise une explication par causes finales est précisément l'apport des mathématiques. La manière dont j'interprète l'extrait consiste à rendre efficace le principe téléologique de moindre action sur la base des acquis des mathématiques et de la physique. Autrement dit, puisqu'on peut en calculer les effets dans les phénomènes observés, le principe de moindre action possède sur les autres principes un critère de validation supplémentaire et justificateur. Ce n'est que rétrospectivement qu'on peut déduire les lois de la nature du principe, mais sa validité théorique prend appui au départ sur des certitudes mathématiques. Par ailleurs, il semble que le terme de déduction employé par Maupertuis à quelques reprises n'ait pas la signification démonstrative et axiomatique qu'on pourrait lui attribuer aujourd'hui. Déduire voudrait dire tirer la validité d'un principe à partir d'un ensemble de constats empiriques et de calculs plutôt que démontrer des conclusions ou des conséquences à partir du principe de moindre action en tant qu'axiome fondamental et évident par soi. Autrement, il serait difficile de donner une interprétation cohérente de la position de Maupertuis ${ }^{21}$. En somme, la validation de ce principe téléologique reposerait essentiellement sur un ensemble de preuves mécaniques et empiriques mathématiquement calculables, et non sur une structure axiomatique et déductive à proprement parler.

3) Pour Maupertuis, l'adoption d'une loi générale téléologique qui détermine les changements dans la nature met le monde dans une relation de dépendance à l'égard de l'Être suprême. Une cause finale régissant les lois de la nature suppose un agent intelligent qui a voulu une telle structure et qui en assure la conformité universelle. Mieux que toute preuve téléologique qui

19. Essai de cosmologie, in Euvres I, p. xiv.

20. Ibid., p. xvii-xxi.

21. L'interprétation que donne Euler du principe de moindre action dans le mémoire qui y est consacré va dans la même direction, en ce que la moindre action est constatée en accord avec tous les phénomènes: Sur le principe de la moindre action, in Histoire, I753, p. 2 I 6. 
cherche un fondement dans les détails de l'organisation du monde ${ }^{22}$, le principe de moindre action démontre sans équivoque l'existence de Dieu, étant donné la nécessité de poser une cause intelligente dans la détermination téléologique des choses. Or il faut aussi insister sur l'apport de ce fondement en Dieu pour le principe de moindre action: non seulement la nature est dans un rapport de dépendance à l'égard de l'Être suprême, mais l'origine divine garantit l'universalité du principe dont il émane:

Notre principe, plus conforme aux idées que nous devons avoir des choses, laisse le Monde dans le besoin continuel de la puissance du Créateur, et est une suite nécessaire de l'emploi le plus sage de cette puissance. Les lois du mouvement ainsi déduites, se trouvant précisément les mêmes qui sont observées dans la Nature, nous pouvons en admirer l'application dans tous les phénomènes, dans le mouvement des animaux, dans la végétation des plantes, dans la révolution des astres; et le spectacle de l'Univers devient bien grand, bien plus beau, bien plus digne de son Auteur ${ }^{23}$.

Certes, la cosmologie de Maupertuis permet l'admiration d'un ordre d'origine divine et renforce par le fait même la croyance en une forme de théisme ${ }^{24}$. Insistons cependant sur l'importance de la portée universelle du principe de moindre action ainsi assurée par voie théologique et métaphysique: une fois établi que la finalité des lois de la nature suppose l'existence de Dieu, car elle ne s'explique pas en termes strictement mécaniques ${ }^{25}$, cette même source divine atteste l'application entière de la moindre action à tous les phénomènes. Les Lettres reviennent d'ailleurs sur ce point: au lieu de proposer un principe qui ne vaut que pour le mouvement des corps élastiques, telle la loi leibnizienne de conservation de la force, le principe de Maupertuis vaut pour tous les types de corps, élastiques ou durs, d'où son universalité sur le plan explicatif ${ }^{26}$. La généralisation de la moindre action de l'optique à toute la physique, défendue dans l'Accord de différentes lois de la nature, irait d'ailleurs dans le même sens ${ }^{27}$. La conséquence scientifique d'une telle universalité est évidemment la possibilité de découvrir, dans les domaines physiques, physiologiques ou astronomiques, les phénomènes en accord avec le principe de moindre action. Dès lors, on trouve en cet endroit une justification supplémentaire à l'élaboration d'une cosmologie centrée autour de la téléologie: une métaphysique de la nature sert à ajuster les théories scientifiques, confirmées par l'expérience et le calcul, à partir d'un

22. Essai de cosmologie, in Euvres I, p. 2I ; Examen philosophique de la preuve de l'existence de Dieu, in Histoire, I758, p. 389-390.

23. Essai de cosmologie, in Euvres I, p. 44-45.

24. Mentionnons également les répercussions de l'existence de Dieu pour la constitution d'une philosophie morale chez Maupertuis: Essai de philosophie morale, in Euvres I, p. 232252.

25. Essai de cosmologie, in Cuvres I, p. 35 .

26. Lettres $X$, in Cuvres II, p. 273.

27. Accord, in Euvres IV, p. $2 \mathrm{I}-22$. 
principe structurant et universel. C'est-à-dire que, en constituant l'assise d'une preuve de l'existence de Dieu, la téléologie aura un impact certes théologique et moral, mais aussi proprement épistémologique et scientifique en ce qu'elle pourrait valider la nature des descriptions physiques qui suivent nécessairement le calcul de la moindre action en tous phénomènes. Le critère d'acceptation ou de rejet d'une théorie, scientifiquement fondée, se trouverait au final dans une conformité générale avec la structure téléologique de la nature fondée en Dieu.

En somme, Maupertuis semble défendre une conception de la cosmologie qui est conforme à la définition donnée en début de présentation: dans un premier temps, la validité d'un principe métaphysique doit se faire en conformité avec les lois et principes des mathématiques et de la physique. La certitude mathématique constituerait ainsi un critère de démarcation entre les différentes lois téléologiques, voire en interdirait la multiplication. Le principe de moindre action n'aurait pas de supériorité sur les autres hypothèses sans une telle légitimation théorique. Dans un deuxième temps, et ce par effet de retour, le fondement universel du principe en Dieu procure à cette explication une fonction incontestable dans l'établissement des théories scientifiques. Sachant que tout se produit avec la plus petite quantité d'action possible, une description physique ne sera en fin de compte acceptable que si elle s'établit sur une telle base métaphysique. La téléologie de Maupertuis a par conséquent un rôle important à jouer dans l'élaboration de la science, bien qu'elle ne semble pas constituer un fondement a priori, premier et théoriquement indépendant, comme c'est le cas par exemple dans la tradition wolffienne. Une métaphysique de la nature ou cosmologie est possible, même nécessaire, mais sans que celle-ci s'inscrive dans un ordre disciplinaire de fondement absolu de la métaphysique sur les théories des sciences de la nature.

\section{La métaphysique eulérienne}

Le positionnement entre métaphysique et physique me semble encore plus évident chez Euler, autre membre influent de l'Académie de Berlin à la même époque. Contrairement à ce que semble croire Maupertuis, le champ d'une métaphysique de la nature ne se réduirait d'ailleurs pas à l'adoption du seul principe de moindre action. À ce sujet, il semble qu'Euler considère de façon analogue que la moindre action est un principe de nature téléologique et que son positionnement par rapport aux différentes sciences suit en bonne partie l'analyse qu'en fournit Maupertuis ${ }^{28}$. Les réflexions métaphysiques d'Euler sont néanmoins plus nombreuses que chez ce dernier, même s'il serait excessif de maintenir qu'elles sont abondantes. La cosmologie eulérienne est plus diversifiée mais reste modeste à bien des égards; l'opposition d'Euler aux

28. À cet égard, voir le texte d'Ansgar Lyssy dans le présent numéro. 
métaphysiques leibnizienne et wolffienne et son adhésion partielle à l'approche newtonienne en philosophie sont très certainement les raisons qui expliquent une certaine forme de scepticisme à l'égard de l'étendue de la métaphysique et de la possibilité de résoudre plusieurs de ses questionnements au delà du domaine de la cosmologie ${ }^{29}$.

Il appert toutefois - et c'est le point sur lequel je désire insister - que le modèle qu'on trouve chez Maupertuis, et qui fait dépendre les hypothèses métaphysiques de la confirmation initiale de certitudes mathématiques et physiques, est similairement présent chez Euler. Premièrement, il semble assez clair que le domaine d'application de la métaphysique, en tout cas celui auquel s'est surtout attardé Euler, est de nature cosmologique. Une métaphysique de la nature recherche quelques principes fondamentaux de la physique quant à la structure et l'ordre des corps matériels. Euler le mentionne dans une lettre à Maupertuis de $\mathrm{I} 745$ où il est question du principe de moindre action et de son rapport à la métaphysique:

En voilà donc une grande science, qui nous manque encore, et qui roule sur les principes généraux qui s'observent dans la nature, et il me semble que c'est là où réside la véritable métaphysique, en tant qu'elle renferme les premiers principes de la physique et de la mathématique; de laquelle la métaphysique de Leibniz et de Wolff est encore bien éloignée ${ }^{30}$.

La critique de la métaphysique d'inspiration leibnizienne et wolffienne est assez courante chez Euler et Maupertuis à la même époque, et je n'entends pas m'y attarder davantage. Retenons pourtant un autre aspect de ce passage qui détermine l'étendue théorique de la métaphysique. Celle-ci permet de découvrir les premiers principes des sciences. On pourrait croire, encore une fois, que les vérités métaphysiques servent de fondement aux sciences, sans lesquelles la physique et même les mathématiques ne sauraient s'établir adéquatement. De sorte qu'il faudrait déterminer les propriétés essentielles de la matière, expliquer l'origine de la force et définir la nature de l'espace et du temps, entre autres choses, pour ensuite élaborer de manière conséquente les lois du mouvement. Or, suivant d'une certaine façon ce que maintient Maupertuis à la même période, Euler ne croit pas qu'une métaphysique de la nature puisse s'instaurer indépendamment des résultats en sciences de la nature. Les réflexions eulériennes sur la cosmologie se font toujours sur la base d'acquis scientifiques, en particulier de la mécanique. Je m'attarderai ici à deux exemples importants dans l'œuvre d'Euler, soit la

29. Les Lettres à une princesse d'Allemagne comportent certes plusieurs réflexions métaphysiques, par exemple sur la question du lien causal entre l'âme et le corps: lettres LXXXLXXXIV. Mais rappelons toutefois qu'il s'agit d'un ouvrage de vulgarisation, dont le contenu et la portée diffèrent par rapport aux autres travaux d'Euler.

30. Lettre à Maupertuis du ıo décembre 1745 , in Euler, Opera omnia, P. Costabel, E. Winter, A. T. Grigorijan et A. P. Juskevic (dir. publ.), Lipsiae/Berolini, Typis et in ædibus B. G. Teubneri; Basel: Birkhäuser Basileae, I9 I I-, 4A, vol. 6, p. 57. 
question de la divisibilité et des moindres parties de la matière, et celle des caractéristiques de l'espace et du temps. Euler a consacré un mémoire à chacune de ces problématiques dont je souhaite analyser les éléments pertinents pour la présente discussion.

\section{Les moindres parties}

Dans un mémoire intitulé Recherches physiques sur la nature des moindres parties de la matière ${ }^{31}$, Euler se pose les questions de savoir si la matière est constituée de particules insécables, mais surtout si celles-ci se différencient les unes des autres par des qualités intrinsèques. Deux positions seraient envisageables: ou bien chaque partie est discernable des autres par sa nature singulière, et cette différenciation se répercuterait sur le plan des corps composés, ou bien les particules sont similaires les unes aux autres quant à leurs caractéristiques particulières, ce qui signifierait que la différenciation se trouve uniquement dans l'arrangement et la figure des corps qui en seraient constitués, et non dans les parties elles-mêmes ${ }^{32}$. Euler convient que ce problème n'est pas résoluble par les moyens de l'expérience et du calcul, mais qu'il s'agit d'un sujet proprement métaphysique:

C'est donc une question bien importante, tant dans la physique que dans la métaphysique, de savoir, si les plus petites particules de la matière sont toutes semblables entre elles, ou non. Les philosophes sont fort partagés sur ce sujet; quelques-uns soutiennent que toutes ces dernières particules diffèrent tellement qu'il n'y en a pas même deux qui soient parfaitement semblables. D'autres veulent au contraire qu'elles se ressemblent toutes parfaitement. Il serait téméraire d'entreprendre la décision de cette question, l'expérience nous refusant tout secours pour cela et la raison seule n'étant pas suffisante pour nous éclaircir sur ce point. Je bornerai donc mes recherches à examiner seulement le rapport qu'il y a entre l'étendue et l'inertie des moindres particules de la matière; car quoiqu'il ne soit pas possible de pousser les expériences jusquelà, j'ai pourtant remarqué que l'expérience commune, aidée de quelques principes incontestables de la raison, nous peut conduire à une conclusion sure, qui ensuite ne manquera pas de nous découvrir plusieurs autres propriétés de la matière, sur lesquelles nous ne sommes que trop incertains $s^{33}$.

31. Seul le résumé du mémoire est paru dans le volume de I 746 de l'Histoire de l'Académie royale des sciences et des belles- lettres de Berlin: p. 28-32. Le texte d'Euler est cependant disponible dans l'édition des Opera omnia à laquelle je me réfèrerai par la suite.

32. Recherches physiques sur la nature des moindres parties de la matière, in Euler, Opera omnia, F. Rudio, A. Krazer, A. Speiser, L. G. Du Pasquier (dir. publ.), 3, vol. I, p. 6-7. Parmi les partisans de la différenciation qualitative des parties de la matière, il faut bien entendu penser à Leibniz et Wolff qui défendent le principe des indiscernables, tandis qu'Euler trouverait déjà en Descartes un défenseur de l'égalité quantitative des parties de l'étendue: AT VIII, p. IOO-IOI.

33. Des moindres parties de la matière, in Opera omnia, p. 7. 
Euler indique explicitement que ni l'expérience ni les principes de la raison ne sont, pris isolément, suffisants pour déterminer l'équivalence ou non des parties de la matière. Il affirme toutefois qu'une solution sera à trouver dans les déterminations d'étendue et d'inertie, connues par ces deux sources comprises ensemble. Plus précisément, Euler se sert du calcul de la gravité spécifique, ou densité, pour savoir si les molécules matérielles sont équivalentes. Mon intention n'est pas ici de reproduire l'argumentaire du texte, mais d'insister sur l'approche qui caractérise la démarche d'Euler. Deux points doivent attirer notre attention: I) la détermination de la nature des parties de la matière ne peut se faire, ni de manière a priori, comme semblent le proposer Leibniz et Wolff, ni par le seul recours à l'expérience; bien entendu il nous est impossible de percevoir par la sensation la composition première des corps, mais uniquement ses arrangements plus grossiers. Néanmoins, une hypothèse acceptable pourra être élaborée autrement. Euler récupère en réalité les deux sources précédentes de connaissance, mais pour les combiner: d'une part, l'expérience commune, dont on pourra tirer des vérités factuelles valides pour la description des moindres parties; d'autre part, certains principes de la raison conduisant à une théorie plus exacte de la matière. Les contributions complémentaires de l'expérience et de la réflexion rationnelle seraient suffisantes pour déterminer une thèse métaphysique; séparées, elles ne sont à l'inverse d'aucun recours. 2) Ces principes de la raison ne semblent cependant pas, pour Euler, être de nature métaphysique. Le résultat de la recherche sera métaphysique, c'est-à-dire qu'on parviendra à décider ontologiquement si les particules matérielles sont qualitativement semblables ou non. Toutefois, les moyens épistémologiques pour y parvenir ne sauraient l'être: les principes dont Euler fait mention sont issus du calcul et de la démonstration géométrique, en particulier la proportionnalité du poids des corps avec l'inertie que Newton a démontrée géométriquement et confirmée empiriquement ${ }^{34}$. Ce qui signifie que la procédure se situe au départ du côté des sciences de la nature, avec l'expérience et le calcul géométrique, pour ensuite arriver à un principe métaphysique, celui de l'équivalence quantitative des gravités spécifiques. Ce premier exemple serait donc en conformité avec le modèle qui fait dépendre les principes métaphysiques d'un travail de théorisation de physique et de mathématique préalable. D'ailleurs, l'affirmation selon laquelle la détermination de la discernabilité des molécules ou parties de la matière ne peut s'expliquer par les seuls principes de la raison, métaphysique ou géométrique, interdit par la même occasion de penser la cosmologie comme fondation des sciences. Un positionnement originel d'une métaphysique de la nature n'est pensable que si elle s'établit, comme chez Wolff et Kant, de manière a priori et première, que ce soit par des moyens proprement ontologiques ou mathématiques. Or Euler ne croit pas qu'une telle autonomie théorique puisse être 
conférée à la cosmologie, car il la place dans une relation de dépendance par rapport aux vérités des sciences de la nature. En somme, la cosmologie eulérienne comprend bel et bien des réflexions métaphysiques sur la structure de la matière, en l'occurrence quant à la thèse qu'il adopte de l'équivalence des masses des particules corporelles, mais pourvu qu'une telle position se base sur les connaissances scientifiques que nous possédons de la nature des corps. Sinon, Euler condamnerait sans hésitation la prétention de déterminer une thèse ontologique sur les constituants matériels, comme il le fait par rapport au principe des indiscernables de Leibniz et de Wolff.

\section{L'espace et le temps}

Une approche très similaire est employée par Euler dans un autre mémoire de l'Académie de Berlin publié en 1750 et intitulé Réflexions sur l'espace et le temps. À nouveau, je ne souhaite pas ici expliciter tout le contenu de l'argumentation eulérienne, mais plutôt souligner une démarche qui ressemble à celle empruntée dans le mémoire précédent sur les moindres parties. Le texte vise à défendre le caractère absolu et réel de l'espace et du temps, en accord avec la position newtonienne, mais en opposition avec la thèse de Leibniz et de Wolff selon laquelle l'espace et le temps sont des déterminations relatives. Comme c'était le cas dans le dernier exemple, Euler convient qu'une réponse à cette question appartient à la métaphysique, et non proprement aux sciences de la nature. Ni les démonstrations géométriques seules ni l'expérience sensible ne fournissent les vérités nécessaires à l'établissement d'une hypothèse métaphysique acceptable quant aux caractéristiques de l'espace et du temps. En l'occurrence, l'expérience sensible est d'autant plus inutile, puisqu'Euler mentionne que l'idée de l'espace, comme celle du temps, n'est pas donnée dans la sensation, mais plutôt dans la réflexion, ce qui signifie que ce ne sont pas des idées données directement par intuition sensible $^{35}$. Parallèlement, une démonstration entièrement géométrique de la réalité absolue de l'espace et du temps n'est en outre pas envisageable. Newton et ses partisans n'y seraient apparemment pas parvenus ${ }^{36}$ et Euler concède donc l'impossibilité d'accomplir une telle tâche. Le seul moyen théorique qui est approprié consiste à se baser sur les lois de la mécanique, qui, elles, sont démontrées géométriquement et confirmées par l'expérience; c'est uniquement à la suite de tels acquis qu'il est possible de déterminer la meilleure hypothèse métaphysique. Euler mentionne surtout les lois du repos et de la directionalité du mouvement fondées sur l'inertie naturelle des corps. Dès le tout début du mémoire, il évoque la solidité des principes de la mécanique,

35. Réflexions sur l'espace et le temps, in Histoire, I750, p. 329.

36. Pensons, par exemple, à la contribution de Joseph Raphson dans son De Spatio reali seu Ente Infinito conamen Mathematico-Metaphysicum de I697 que Luc Petershmitt a récemment commenté et traduit: Peterschmitt, 20I4, p. I97-22I. 
dit autrement leur indépendance théorique, bien qu'il ne soit pas possible d'en démontrer le fondement par voie métaphysique:

Les principes de la Mécanique sont déjà si solidement établis qu'on aurait grand tort si l'on voulait encore douter de leur vérité. Quand même on ne serait pas en état de les démontrer par les principes généraux de la Métaphysique, le merveilleux accord de toutes les conclusions, qu'on en tire par le moyen du calcul, avec tous les mouvements des corps tant solides que fluides sur la terre, et même avec les mouvements des corps célestes, serait suffisant pour mettre leur vérité hors de doute ${ }^{37}$.

Par conséquent, même si la certitude des lois de la mécanique n'est pas métaphysique, ces lois sont suffisamment validées dans la physique pour pouvoir être considérées comme exprimant la nature des déterminations des corps. Or c'est justement cette certitude de la mécanique qui pourra servir de levier à la détermination du problème métaphysique de la réalité de l'espace et du temps. La supériorité d'une hypothèse sur une autre, l'absolutisme newtonien ou le relativisme leibnizien s'évaluera à sa conformité avec les vérités physiques dont la certitude est dorénavant admise. La discrimination entre différents principes métaphysiques se fait à la lumière d'un accord avec la mécanique, dont les lois du repos et du mouvement sont les principaux fondements :

Ces deux vérités étant si indubitablement constatées, il faut absolument qu'elles soient fondées dans la nature des corps: et comme c'est la Métaphysique qui s'occupe à rechercher la nature et les propriétés des corps, la connaissance de ces vérités pourra servir de guide dans ces recherches épineuses. [...] C'est donc toujours une grande avance, quand on connaît déjà d'ailleurs quelques conclusions, auxquelles les premiers principes de la Métaphysique doivent aboutir: et ce sera sur ces conclusions qu'il faudra régler et déterminer les premières idées de la Métaphysique ${ }^{38}$.

Il s'agit sans aucun doute d'un des passages du corpus eulérien qui est le plus clair quant à l'articulation théorique entre physique et métaphysique. L'intention d'Euler est manifeste: l'adoption de vérités de type physique servira de guide dans les recherches laborieuses et hors de la portée des outils proprement scientifiques que sont l'observation et le calcul. Autrement dit, il est difficilement concevable, voire impossible de parvenir à des principes métaphysiques, dans ce cas-ci quant à la réalité de l'espace et du temps, sans s'appuyer sur les résultats certains de la mécanique. La relation entre les deux genres de principes est sans équivoque: sans les sciences de la nature, la métaphysique ne saurait s'établir sur des bases appropriées. Comme Euler le maintenait dans le mémoire sur les moindres parties de la matière, la mesure d'une vérité cosmologique exige des lois et vérités physiques préalables sans

37. Réflexions sur l'espace et le temps, in Histoire, I750, p. 324.

38. Ibid., p. 324-325. 
lesquelles on demeure à un niveau trop grand d'incertitude. Ce qui veut dire que non seulement Euler partage avec Maupertuis une même conception de la métaphysique de la nature, en ce qu'elle dépend des recherches mathématiques et physiques - et non l'inverse, mais il semble aussi qu'il convienne avec lui de la manière de légitimer une entreprise cosmologique. Renverser le rapport de priorité classique entre métaphysique et physique est le seul moyen de sauvegarder la validité des principes ontologiques.

Dans ce contexte, il est vrai qu'on pourrait faire l'objection suivante: si la métaphysique n'a pas pour but de fonder les sciences de la nature, à quoi pourrait-elle servir? Par exemple, une théorie des monades et des éléments dans les philosophies de Leibniz et de Wolff sert notamment à déterminer la nature des corps sur lesquels portent les sciences naturelles et, bien entendu, d'après ceux-ci, à produire des théories scientifiques plus adéquates $^{39}$. Si une métaphysique n'aide plus à fonder la physique, quelle pourrait donc être son utilité ? Or, pour Euler, à la manière de Maupertuis, cette justification de la métaphysique de la nature conserve quand même une légitimité épistémologique, malgré son repositionnement par rapport aux sciences de la nature: la cosmologie, qui fournit les principes premiers de la nature, aura des répercussions rétroactives sur l'élaboration et la complétude des théories scientifiques. On se souvient que la caractérisation téléologique du principe de moindre action permettait à Maupertuis d'en universaliser la portée: que l'on conduise des recherches en optique, en astronomie, en mécanique ou en physiologie, il faudra tenir pour acquis que la nature produit dans ces différents phénomènes toujours le moins d'action possible, selon un principe métaphysiquement prouvé. Une hypothèse ou une théorie scientifique qui stipulerait le contraire serait par conséquent à écarter. Euler semble d'accord sur ce point dans l'emploi des thèses cosmologiques: par exemple, en soutenant la réalité de l'espace, on confère à la géométrie, employée dans la mécanique, un fondement réel. L'espace est ainsi non pas une entité imaginaire, comme le conçoivent à ses yeux Leibniz et Wolff, mais un être réel, ce qui justifie par le fait même la correspondance entre les calculs géométriques et les déterminations réelles des corps dans l'espace absolu ${ }^{40}$. Autrement, il serait difficile de justifier l'usage de la géométrie en physique. Par un effet de retour qu'admettait également Maupertuis relativement au principe de moindre action, l'admission de principes métaphysiques se répercute sur la validité et la portée des sciences physiques, en l'occurrence quant à la justesse de la géométrie comme discipline

39. On pourrait notamment penser au rôle que confère Leibniz au questionnement métaphysique dans un texte publié en I7 I 8 et bien connu au XVIII ${ }^{\mathrm{e}}$ siècle, soit les Principes de la nature et de la grâce. Par exemple, la métaphysique sert à donner la raison du mouvement, sur la base du principe de raison suffisante, puisque la nature en elle-même est indifférente à tel mouvement ou repos de la matière: Die philosophischen Schriften von G. W. Leibniz, hrsg. von Gerhardt, Hildesheim, Olms, I965, VI, p. 602.

40. Réflexions sur l'espace et le temps, in Histoire, I750, p. 326. 
mesurant les quantités réelles de l'espace. Bien que la métaphysique de la nature ne possède pas l'autonomie théorique que lui conféraient certains auteurs de la tradition et ne puisse constituer un fondement pour les théories scientifiques, Maupertuis et Euler lui attribuent quand même une fonction importante dans le perfectionnement et la validation de la connaissance scientifique.

\section{Conclusion}

Les conceptions de Maupertuis et d'Euler quant à la place de la métaphysique de la nature, qui sont à mon avis assez convergentes, ne sont toutefois pas représentatives de la richesse des positions maintenues par les membres de l'Académie de Berlin au milieu du XviII ${ }^{\mathrm{e}}$ siècle. J'ai déjà cité l'exemple de d'Alembert qui récupère une position similaire à celle de Condillac. Sa condamnation de la cosmologie irait de pair avec la réduction du champ métaphysique aux seuls questionnements psychologiques sur les sources et les facultés de la connaissance humaine. Dans le camp de ceux qui défendent plutôt une définition fondationaliste de l'ontologie, à la manière de Descartes et de Wolff, on peut citer Formey, secrétaire de l'Académie qui reste à bien des égards assez fidèle au wolffisme ${ }^{41}$. D'autres membres importants seraient d'un avis similaire, même s'ils défendent des thèses métaphysiques ou épistémologiques qui s'écartent très souvent de l'orthodoxie wolffienne. C'est notamment le cas de Mérian. Dans un discours publié en 1767 , intitulé Discours sur la métaphysique, celui-ci récupère une définition beaucoup plus classique de la métaphysique et de sa division entre théologie, cosmologie et psychologie ${ }^{42}$. Bien que Mérian reconnaisse aux sciences, en particulier à la physique, une certaine autonomie théorique, il est d'avis que c'est à la métaphysique d'en donner les premiers fondements:

Il ne faut qu'être de bonne foi pour convenir que la Métaphysique s'attache à de plus hauts objets que les sciences naturelles. Elle pénètre dans l'intérieur des choses dont la Physique ne fait qu'entamer l'écorce. [...] La Physique ne tire-t-elle pas son plus grand lustre du jour qu'elle répand sur la Théologie naturelle? N'est-ce pas là le lieu commun que ses panégyristes ont le plus souvent rebattu? Mais la Physique, à cet égard, ne donne que des faits; c'est à la Métaphysique à les convertir en preuves ${ }^{43}$.

De cette manière, Mérian ne remet en question ni le contenu traditionnel de la métaphysique et de ses sous-disciplines, ni le positionnement de cette science première par rapport aux autres savoirs. Seule la métaphysique permet d'expliquer l'intérieur des choses et de donner les fondements nécessaires aux sciences. Il est vrai que ce texte ne constitue pas un mémoire de

41. Par exemple dans son ouvrage le plus connu: Johann Henrich Samuel Formey, La Belle Wolfienne, La Haye, Le Vier, I74I, I, p. 3 I.

42. Discours sur la métaphysique, in Histoire, $1767, \mathrm{p} .452$.

43. Ibid, p. 454-455. 
nature savante, mais un discours public dans lequel Mérian adopte vraisemblablement une conception encore répandue de la discipline. Il n'empêche que ce texte est l'occasion de réitérer certaines idées quant à la portée et l'objet de la métaphysique qui semblaient toujours d'actualité à son auteur. En ce qui concerne Nicolas de Béguelin, qui s'intéressa également à ces questions, il est peut-être plus près de Maupertuis et d'Euler: dans deux mémoires intitulés Sur les premiers principes de la métaphysique, il soutient que les principes métaphysiques sont ceux auxquels toutes les autres sciences devraient aboutir ${ }^{44}$, ce qui n'est pas éloigné du sentiment d'Euler souligné précédemment. Béguelin est toutefois beaucoup plus ouvert à des réflexions sur les objets généraux de la métaphysique, notamment l'articulation entre les principes de contradiction et de raison suffisante, ce que se refuseraient à maintenir aussi bien Maupertuis qu'Euler ${ }^{45}$.

Dans le présent article, il ne s'agissait toutefois pas de dresser un portrait de la situation de la métaphysique, en particulier de la métaphysique de la nature, à l'Académie de Berlin des années I740 à I760, mais de marquer une tendance, celle empruntée par Maupertuis et Euler, qui propose une conception particulière et assez originale de la cosmologie. L'approche théorique et le contenu de la métaphysique de la nature divergent chez l'un et chez l'autre, mais les deux penseurs semblent se rejoindre sur deux points importants: premièrement, la validité des principes métaphysiques repose nécessairement sur les acquis des mathématiques et de la physique. Sans guide pour déterminer les hypothèses les plus acceptables, la métaphysique semble condamnée à en rester à des spéculations creuses et sans fondements. L'une des conséquences de cette conception est que la métaphysique ne peut plus être considérée comme étant première par rapport aux autres sciences physiques. Maupertuis et Euler opéreraient donc un renversement par rapport à l'interprétation classique du positionnement entre métaphysique et physique qu'on trouve chez Descartes, Wolff ou Kant, mais aussi chez certains de leurs collègues comme Formey et même Mérian. Deuxièmement, la légitimité du discours métaphysique s'évalue à ses répercussions rétroactives sur les sciences. Les principes de la métaphysique, s'appuyant sur ceux de la physique, pourront servir à mieux déterminer la portée et l'objet des sciences. Dans un contexte théorique où la métaphysique de la nature est de plus en plus remise en question, le rôle qu'elle y joue en dépend. L'utilité de la cosmologie se comprendrait somme toute à la lumière de ses retombées sur les différentes sciences de la nature.

44. Sur les premiers principes de la métaphysique, in Histoire, I757, p. 408.

45. Sur ces aspects de la philosophie de Béguelin et son projet conciliateur, voir l'article de François Duchesneau dans le présent numéro. 